patients and controls. Factors associated with sero-reversion of the anti-HPV antibodies were studied by statistical analyses.

Results: 50 SLE patients (age $25.8 \pm 3.9$ years) and 50 controls $(25.8 \pm 3.9$ years) were vaccinated with Gardasil in 2011. The sero-conversion rates of anti-HPV serotypes $6,11,16$ and 18 in patients and controls were $82 \%, 89 \%, 95 \%, 76 \%$, and $98 \%, 98 \%, 98 \%, 80 \%$, respectively, at month 12 post-vaccination. Among those subjects who sero-converted and were available for follow-up, persistence of the antibodies to HPV serotypes $6,11,16$ and 18 at 5 years was present in 24/ 27 (89\%), 26/31 (84\%), 32/34 (94\%), 24/25 (96\%) of the SLE patients and 32/33 $(97 \%), 32 / 33(97 \%), 32 / 32(100 \%)$ and $23 / 24(96 \%)$ of the controls, respectively. Moreover, antibody titers to HPV serotypes 6 and 16 at 5 years were significantly lower in SLE patients than controls. Seven (21\%) SLE patients had sero-reversion of any one of the four anti-HPV antibodies $(6,11,16$ or 18$)$ at year 5 post-vaccination. Patients who sero-reverted had received a significantly higher cumulative dose of prednisolone $(13.0 \pm 5.34$ vs $4.63 \pm 4.59$ grams; $p=0.002)$, mycophenolate mofetil ( $1050 \pm 1180$ vs $238 \pm 49.5$ grams; $p=0.007)$ and tacrolimus $(375 \pm 580$ vs $268 \pm 1180 \mathrm{mg} ; \mathrm{p}=0.03$ ) during the 5 year follow-up than those with persistence of immunogenicity. These sero-reverted patients had also received a non-significantly higher cumulative dose of cyclophosphamide $(1.97 \pm 5.22$ vs $0.43 \pm 2.24$ grams; $p=0.27$ ). In addition, sero-reverted patients had more SLE flares in the 5 year follow-up period compared to those with persistence of immunogenicity (3.14 \pm 1.21 vs $1.89 \pm 1.28 ; p=0.03$ ). Among 64 flares in patients with persistent anti-HPV antibodies and 26 flares in those with sero-reversion, renal flares occurred more frequently in the latter group of patients $(16 \%$ vs $38 \% ; p=0.02)$.

Conclusions: Immunogenicity of the quadrivalent HPV vaccine was retained in $79 \%$ of SLE patients at 5 year post-vaccination. Antibody titers to HPV serotypes 6 and 16 were significantly lower in SLE patients than controls. Patients who had more SLE flares, especially renal flares, and had received higher cumulative doses of glucocorticoids, mycophenolate mofetil and tacrolimus were more likely to have sero-reversion of one or more anti-HPV antibodies. Re-vaccination of these patients should be considered.

Disclosure of Interest: None declared

DOI: 10.1136/annrheumdis-2018-eular.4332

\section{SAT0422 INDIRECT PATHOGENIC ROLES OF CEREBROSPINAL FLUID ANTI-U1RNP ANTIBODIES IN THE PRESENCE OF ANTI-NR2 ANTIBODIES IN PATIENTS WITH NEUROPSYCHIATRIC SYSTEMIC LUPUS ERYTHEMATOSUS}

T. Fujii $^{1,2}$, S. Kondo-Ishikawa ${ }^{3}$, N. Ishigooka ${ }^{2}$, H. Yoshifuji ${ }^{2}$, K. Ohmura ${ }^{2}$, T. Mimori ${ }^{2} .{ }^{1}$ Rheumatology and Clinical Immunology, Wakayama Medical University, Wakayama; ${ }^{2}$ Rheumatology and Clinical Immunology, Kyoto University School of Medicine; ${ }^{3}$ Rheumatology, National Hospital Organization Utano Hospital, Kyoto, Japan

Background: Autoantibodies (auto Abs) and inflammatory mediators (IMs) in cerebrospinal fluid (CSF) may be involved in the pathogenesis of neuropsychiatric systemic lupus erythematosus (NPSLE). Previous studies indicated that anti-Nmethyl $d$-aspartate receptor NR2 subunit (NR2) Abs have a pathogenic role in NPSLE by BBB abruption. Hirohata S, et al, Arthritis Res Ther, $2014 \mathrm{We}$ also reported the association between NPSLE and CSF-anti-U1RNP Abs (Sato, et al, $A$ and $R$, 2010).

Objectives: In the present study, synergistic effects of these auto Abs, their associated IMs, and blood brain barrier (BBB) permeability are determined in NPSLE patients.

Methods: CSF samples were collected from 69 of NPSLE patients at their acute phase and 13 of non-NPSLE control after obtaining the written informed consent. CSF anti-NR2 and anti-U1RNP Abs were determined by ELISA. CSF IL-6, IL-8, and monokine induced by IFN- $\gamma$ (MIG) were measured by quantitative multiplex cytokine analysis. BBB permeability was evaluated by albumin quotient (Qalb).

Results: 1) CSF IL-6 levels were higher in CSF anti-NR2 Ab +ve $(70 \pm 178 \mathrm{pg} / \mathrm{mL})$ than in -ve $(11 \pm 32, p=0.02)$ patients and in controls $(16 \pm 40, p=0.003)$ and positively correlated with anti-NR2 Ab titer $(r=0.42, p=0.003)$. Anti-U1RNP Ab positivity was not associated with CSF IL6 elevation. 2) CSF IL-8 levels were higher in CSF anti-U1RNP Ab +ve than in -ve $(270 \pm 862$ vs. $52 \pm 177 \mathrm{pg} / \mathrm{mL}, p=0.04)$ patients. Anti-NR2 Ab positivity was not involved in CSF IL8 elevation. 3) CSF MIG levels were more elevated in both CSF anti-NR2 +ve $(4483 \pm 11845 \mathrm{pg} / \mathrm{mL}$, $\mathrm{p}=0.04)$ and anti-U1RNP Ab +ve $(5519 \pm 13504, \mathrm{p}=0.03)$ patients than in controls $(115 \pm 125)$. 4) All the patients were divided into 4 groups: CSF anti-NR2 +ve/antiU1RNP+ve (double positive [DP], $n=9$ ), anti-NR2 +ve/anti-U1RNP -ve (aNR2, $\mathrm{n}=15)$, anti-NR2 -ve/anti-U1RNP+ve (aU1RNP, $\mathrm{n}=9$ ), and anti-NR2 -ve/anti-
U1RNP -ve (double negative [DN], $\mathrm{n}=36$ ). In comparison between DP and aNR2 groups, anti-U1RNP Abs had a synergistic effect on CSF IL-6 elevation (150 \pm 260 vs. $18 \pm 44 \mathrm{pg} / \mathrm{mL}, \mathrm{p}=0.03$ ) that is not directly associated with anti-U1RNP Ab positivity. In comparison with aNR2 group, both CSF IL-8 (543 \pm 1198 vs. $43 \pm 102$ pg/ $\mathrm{mL}, \mathrm{p}=0.04)$ and MIG (10104 \pm 17748 vs. $1111 \pm 2584 \mathrm{pg} / \mathrm{mL}, \mathrm{p}=0.03$ ) levels which were positively correlated with CSF anti-U1RNP Ab titer, were more elevated in DP group. 5) Qalb was positively correlated with CSF IL-8 ( $r=0.51$ $\mathrm{p}<0.0001)$ and MIG $(r=0.44, \mathrm{p}=0.0003)$, suggesting that CSF anti-U1RNP Ab pos itivity is involved in the BBB abruption. Actually, Qalb $\left(\times 10^{3}\right)$ tended to be higher in DP than in aNR2 group $(13 \pm 9$ vs. $9 \pm 8, p=0.06)$ and CSF anti-NR2 Ab titer appeared to be more elevated in DP than in aNR2 group ( $81 \pm 47$ vs. $57 \pm 21$, $\mathrm{p}=0.06)$.

Conclusions: The present study suggested that anti-NR2 and U1RNP Abs have synergistic effects on CSF IL-6 elevation in patients with NPSLE. CSF IL-6 level was associated with anti-NR2 Ab titers, which depend on BBB permeability. CSF anti-U1RNP Ab-mediated IL-8 and MIG elevation may induce BBB abruption followed by anti-NR2 Ab penetration into CSF.

\section{REFERENCES}

[1] Sato T, Fujii T, et al. Arthritis Rheum 2010;62:3730.

[2] Yokoyama T, Fujii T, et al. Lupus 2014;23:635.

Acknowledgements: The present study is supported by the Japanese Ministry of Education, Culture, Sports, Science, and Technology.

Disclosure of Interest: None declared

DOI: 10.1136/annrheumdis-2018-eular.3033

\section{SAT0423 MOLECULAR PROFILES ASSOCIATE WITH CLINICAL DISEASE ACTIVITY AND INFORM PATIENT SUBSETTING IN ADULT SYSTEMIC LUPUS ERYTHEMATOSUS}

J.M. Guthridge, R. Lu, C. Arriens, T. Aberle, S. Kamp, M.E. Munroe, T. Gross, W. DeJager, S.R. Macwana, R.L. Bourn, S. Apel, H. Chen, E.F. Chakravarty, A. Thanou, J.T. Merrill, J.A. James. Arthritis and Clinical Immunology, Oklahoma Medical Research Foundation, Oklahoma City, USA

Background: Systemic lupus erythematosus (SLE) is characterised by remark able clinical and pathophysiological diversity, hindering diagnosis, treatment and treatment development. Subsetting of patients based upon clinical presentations alone has not identified homogeneous groups of patients best treated with a directed therapeutic.

Objectives: To cluster SLE patients into more homogeneous subsets with common molecular pathway signatures, and to assess the clinical, therapeutic, and demographic features enriched in each cluster.

Methods: Serial or single plasma, serum and RNA samples $(n=290)$ were collected from 198 SLE patients who met ACR classification. Disease activity was assessed by modified SELENA-SLEDAI at an average of 17 visits per patient. Transcriptional co-expression signature module scores were calculated from Illumina Beadchip Microarray gene expression data for 29 immune pathway related modules. Plasma soluble mediators $(n=23)$ and 12 antinuclear autoantibodies (anti-dsDNA, chromatin, ribosomal P, Sm, Sm, SmRNP, RNP, Ro/SSA, La/SSB centromere B, Scl-70 and Jo-1) were assessed by multiplex bead-based assay and ELISA. Spearman correlations were used for univariate and multivariate analysis using R. Patients were clustered on module signature scores and soluble mediators using random forest and tSNE.

Results: SLEDAI scores strongly correlated with interferon modules and were modestly correlated with plasmablast and select cell cycle signatures in this adult lupus collection. SLEDAI scores also correlated with soluble levels of IFNa, IL21, IL1a, IL17A, IP10 and MIG. Random forest defined seven clusters of SLE patients with unique molecular phenotypes based upon gene co-expression module signatures and soluble mediators. Inflammation and interferon (IFN) signatures were elevated in Clusters 1 (moderately) and 4, with decreased $\mathrm{T}$ cell signatures in Cluster 4. The other clusters had lower IFN and inflammation signatures, but differed in their monocyte, plasmablast and T cell signatures. Clusters 1 and 4 had the highest SLEDAI scores, with high rates of anti-dsDNA, low complement, proteinuria and hematuria; these features were also prominent in Cluster 3 , which lacked the IFN and inflammation signatures. Cluster 6 had the highest plasmablast module score, highest IL1a levels, and SLEDAI scored rashes, but only moderate IFN and inflammation module scores. Cluster 2 had higher rates of SLEDAI scored alopecia, a slightly elevated inflammation signature, but lower interferon signature and lower soluble mediator levels. 


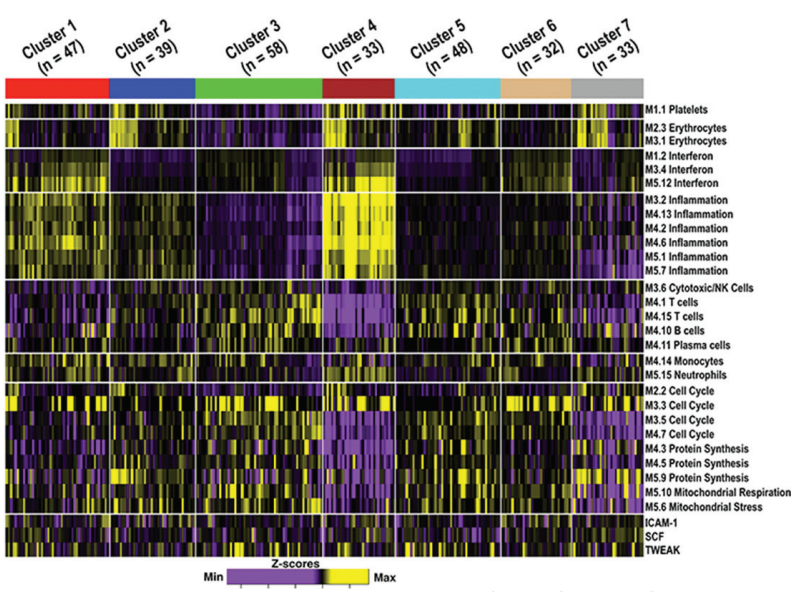

Abstract SAT0423 - Figure 1. Modular transcriptional score profiles of seven SLE clusters

Conclusions: SLE subsets can be distinguished by a range of molecular profiles encompassing IFN, T cell, neutrophil, plasmablast, and inflammation co-expression signatures, as well as soluble mediators that vary with disease activity. Prospective longitudinal studies of these molecular profiles may inform clinical trial design and personalised disease management.

Acknowledgements: This work was supported in part by grants from the National Institutes of Health: U19AI082714, U01Al101934, U54GM104938, and P30AR053483.

Disclosure of Interest: None declared

DOI: 10.1136/annrheumdis-2018-eular.6054

\section{SAT0424 COMPLEMENT C4 GENE COPY NUMBER VARIATIONS BESTOW LARGE RANGES OF SERUM C4 PROTEIN LEVELS IN CHINESE PATIENTS WITH SYSTEMIC LUPUS ERYTHEMATOSUS (SLE) AND CONTRIBUTE TO ORGAN AND CARDIOVASCULAR DAMAGES OVER TIME}

C.C. Mok ${ }^{1}$, E. King ${ }^{2}$, B. Zhou ${ }^{2}$, G. Yu ${ }^{2}$, Y.L. $\mathrm{Wu}^{2}$, C.Y. Yu ${ }^{2} .{ }^{1}$ Medicine, Tuen Mun Hospital, HK, Hong Kong, ${ }^{2}$ Pediatrics, Nationwide Children's Hospital, Columbus, Ohio, USA

Background: Human SLE is characterised by fluctuating serum levels of complement proteins. There are frequent copy number variations (CNVs) of complement $\mathrm{C} 4 \mathrm{~A}$ and $\mathrm{C} 4 \mathrm{~B}$ genes among different individuals. Previously, we demonstrated that $\mathrm{C} 4 \mathrm{~A}$ deficiency is a strong genetic risk factor for SLE.

Objectives: To investigate how CNVs of $\mathrm{C} 4$ contribute to the great variability of $\mathrm{C} 4$ serum levels and how deficiencies of $\mathrm{C} 4 \mathrm{~A}$ or $\mathrm{C} 4 \mathrm{~B}$ modulate the clinical presentations, including organ damage, of SLE.

Methods: Our study population included 499 patients from Hong Kong, who fulfilled $\geq 4$ of the 2013 ACR/SLICC criteria for SLE. Among them $93 \%$ were women, the mean age of SLE onset was $32.8 \pm 13.0$ years, and SLE duration was $14.4 \pm 7.6$ years. Gene copy numbers (GCNs) of total C4 (C4T), C4A and C4B were determined by real-time PCRs. Serial serum levels over the past 5 years for C4 and C3 of each patient were retrieved through the laboratory data registry system. Serum C4 and C3 levels are shown as mg/100 ml (unit). Clinical manifestations and organ damage of SLE were correlated with CNVs of C4 genes and serum levels. Continuous data between groups were compared by t-tests and categorical data by $\chi 2$ analyses. Logistic regression was used to calculate odds ratios (ORs) and 95\% confidence intervals for effects of C4 CNVs on cumulative clinical manifestations of SLE and accrued organ damage, adjusted for durations of disease.

Results: Serum levels for C4 varied from 1-84 units (Median: 17) and for C3 from 8-314 units (Median: 86). There was a very strong correlation between C4 and C3 protein levels $\left(R=0.70, p=5.3 \times 10-{ }^{75}\right)$. The GCN of C4T varied between 2 and 9 with a median of 4 copies (54\%), followed by 2 and 3 copies $(21 \%)$. Each additional gene copy correlated to an increase of 4 and 6 units for the mean and maximum serum C4 levels, respectively. A higher GCN of C4T ( $\geq 3$ vs $<3$ ) was protective against the development of neuropsychiatric disorder over time [OR $0.45(0.21-0.98), p=0.04]$. A high GCN of C4L ( $\geq 3$ vs $<3)$, or the absence of C4S $(\mathrm{GCN}=0)$, was negatively associated with the occurrence of thrombocytopenia
[OR $0.64(0.42-0.97), p=0.04]$. A high GCN of C4B was associated with damage to any organ [OR 1.76 (1.05-2.93), $\mathrm{p}=0.03$ ], but a high $\mathrm{GCN}$ of $\mathrm{C} 4 \mathrm{~A}(\geq 3$ vs $<3$ ) was associated with cardiovascular damage [OR $2.30(1.06-5.00), p=0.04]$. Among the SLE patients studied, $18.3 \%$ had persistently low levels of C4 (mean $\leq 10.0$ units). These patients mostly had $\mathrm{GCNs}$ of $\mathrm{C} 4 \mathrm{~T}=2$ or 3 [OR 4.02 (2.47-6.56), $\mathrm{p}=4.7 \times 10^{-8}$ ], or $\mathrm{C} 4 \mathrm{~B}=0$ or 1 [OR $3.06(1.89-4.96), \mathrm{p}=9.0 \times 10^{-6}$ ]. Patients with persistently low $\mathrm{C} 4$ levels had increased prevalence of mucosal ulceration [OR 2.09 (1.15-3.78), $\mathrm{p}=0.02$ ], lymphopenia [OR 1.76 (1.01-3.05), $p=0.045$ ] and gastrointestinal disorders [OR $2.52(1.31-4.84), p=0.005]$.

Conclusions: CNVs of C4 genes confer great variability of serum C4 levels among SLE patients. While C4A deficiency contributes to genetic predisposition of SLE, persistently low levels of serum C4 among patients were strongly correlated with low GCN of total C4 and C4B deficiency. Elucidating C4-CNVs may have prognostic significance of SLE as high GCNs of C4B and C4A appeared to correlate with organ damage and cardiovascular disease, respectively.

Disclosure of Interest: None declared DOI: 10.1136/annrheumdis-2018-eular.4974

\section{SAT0425 PHENOTYPING PRIMARY SJÖGREN'S SYNDROME (PSS) BY USING SALIVARY GLAND ULTRASONOGRAPHY: A STEP FORWARD TO INDIVIDUALISED THERAPIES}

C. Baldini ${ }^{1}$, F. Ferro ${ }^{1}$, N. Luciano ${ }^{1}$, E. Calabresi ${ }^{1}$, E. Elefante ${ }^{1}$, V. Donati ${ }^{2}$, M. Mosca ${ }^{1}$, S. Bombardieri ${ }^{1} .{ }^{1}$ Rheumatology Unit, University of Pisa; ${ }^{2}$ Pathologic Anatomy II, Pisa, Italy

Background: Salivary gland ultrasonography (SGUS) has recently appeared as a useful tool to non invasively assess major salivary glands inflammation and damage in primary Sjögren's syndrome (pSS). To date, relatively few studies have evaluated the usefulness of SGUS in characterising different disease phenotypes ultimately providing information on which patients might be most likely to benefit from specific interventions.

Objectives: to explore whether SGUS may be useful in identifying different clinical-serological and histopathological phenotypes of pSS, ultimately allowing individualised treatments.

Methods: Sonographic data were retrieved from a large monocentric cohort of pSS patients. The echostructure of each gland on B-mode images was graded on a 5-point scale (0-4), and a SGUS score $\geq 2$ was defined as pathological. Hypoanechoic areas in the glands were defined as isolated $(<25 \%$ of the surface), localised $(25 \%-50 \%)$ and diffuse-scattered ( $>50 \%)$. The biggest hypo or anechoic area in each gland was measured. SGUS was repeated in 150 pSS patients at least twice over a median follow up of 24 months. All the patients received a complete rheumatological assessment: disease activity was measured by the EULAR Sjögren's Syndrome Disease Activity Index (ESSDAI), histopathological evaluation included the assessment of the focus score (FS) and the reporting of the number of germinal centre (GC)-like structures.

Results: We included in this study 311 patients $(300 \mathrm{~F}: 11 \mathrm{M}$; median age $=58$ years (IQR, 49-69)) and median disease duration of 3 years (IQR 0-9). Out of them $136 / 311(43.7 \%)$ had a SGUS score $<2$ with none or isolated hypo-anechoic areas in their glands. Localised areas were detected in $87 / 311$ (28\%) pSS patients and diffuse-scattered areas in $88 / 311$ (28.3\%). Patients with none or isolated hypo-anechoic areas tended to be older $(p=0.06)$ and presented a significantly lower FS, a lower ESSDAI and a higher unstimulated salivary flow rate when compared to the other pSS patients $(p<0.001)$. Patients with localised or diffuse-scattered echographic pattern were more frequently seropositive for anti-Ro/SSA, anti-La/SSB and rheumatoid factor, presented more frequently hypergammaglobulinemia and low C3/C4 levels and had a higher frequency of GC-like structures in their biopsies $(p<0.001)$. Patients with diffuse-scattered hypo-anechoic areas presented the highest disease activity, in particular in the ESSDAI glandular, cutaneous and haematological domains. Notably, the SGUS score did not change over the time in patients who underwent repeated SGUS evaluations. Out of 136 patients with a SGUS score $<2,58$ underwent additional SGUS evaluations over the follow-up. The SGUS was unchanged in all the cases and still associated with a low systemic disease activity.

Conclusions: This study highlighted the potential usefulness of SGUS in phenotyping pSS subsets. Patients with none or isolated hypoechoic areas in their glands are likely to present a low systemic disease activity during the disease course, being therefore candidate to less aggressive treatments. By contrast, patients with highest SGUS scores tend to present a more active systemic involvement and are candidate to receive a more aggressive therapy.

Disclosure of Interest: None declared

DOI: 10.1136/annrheumdis-2018-eular.5948 